\title{
Brown hyaenas on roads: Estimating carnivore occupancy and abundance using spatially auto-correlated sign survey replicates
}

\author{
Michelle Thorn ${ }^{\mathrm{a}, \mathrm{b}, *}$, Matthew Green ${ }^{\mathrm{b}}$, Philip W. Bateman ${ }^{\mathrm{b}}$, Stephen Waite ${ }^{\mathrm{c}}$, Dawn M. Scott ${ }^{\mathrm{a}, 1}$ \\ ${ }^{a}$ University of Brighton, Biology Division, Huxley Building, Lewes Road, Moulsecoomb, Brighton BN2 4GJ, UK \\ ${ }^{\mathrm{b}}$ Mammal Research Institute $\mathcal{E}$ Centre for Wildlife Management, University of Pretoria, Private Bag 0002, Pretoria, South Africa \\ ${ }^{\mathrm{c}}$ Hartpury College, UWE, Hartpury, GL19 3BE, UK
}

\section{A R T I C L E I N F O}

\section{Article history:}

Received 18 October 2010

Received in revised form 18 February 2011

Accepted 12 March 2011

Available online $\mathrm{xxxx}$

\section{Keywords:}

Attitude

Detection probability

Hyaena brunnea

Interview

Wildlife monitoring

South Africa

\begin{abstract}
A B S T R A C T
Carnivore survey protocols that properly address spatial sampling and detectability issues are seldom feasible at a landscape-scale. This limits knowledge of large-scale patterns in distribution, abundance and their underlying determinants, hindering conservation of globally threatened carnivore populations. Occupancy analysis of data from logistically efficient sign surveys along consecutive road segments (spatially auto-correlated replicates) offers a potential solution. We adapted and applied this newly-developed method over $62,979 \mathrm{~km}^{2}$ of human-modified land in South Africa. Our aims were to (1) generate unbiased estimates of brown hyaena occupancy and abundance (2) investigate two suspected determinants of occupancy using a combination of biological and socio-economic sampling techniques, and (3) use simulations to evaluate the effort required for abundance and occupancy estimates with acceptable bias, precision and power. Brown hyaena occupancy was estimated at 0.748 ( \pm SE 0.1 ), and estimated overall density in agricultural land $\left(0.15 / 100 \mathrm{~km}^{2}, \pm S E 0.08\right)$ was an order of magnitude lower than in protected areas. Positive attitudes to carnivores and presence of wildlife farms exerted strong positive effects on occupancy, so changes in these factors may well exert monotonic impacts on local metapopulation status. Producing reliable occupancy and abundance estimates would require $\geqslant 6$ replicates and $\geqslant 12$ replicates per site respectively. Detecting 50\% and 30\% declines in brown hyaena occupancy with adequate power would require five annual surveys at $\geqslant 65$ sites and $\geqslant 125$ sites respectively. Our results suggest that protocols based on spatially auto-correlated sign survey replicates could be used to monitor carnivore populations at large, and possibly even country-wide spatial scales.
\end{abstract}

() 2011 Elsevier Ltd. All rights reserved.

\section{Introduction}

Finding efficient and practical ways to survey carnivores throughout their ranges is of increasing urgency in view of global population declines and mounting threats (Inskip and Zimmermann, 2009; Treves and Karanth, 2003). However, carnivores are inherently elusive, so failure to detect them when present is a common source of survey bias (Karanth and Nichols, 2002; Linkie et al., 2007). Furthermore, heterogeneous detectability confounds inference of spatial and temporal patterns unless properly accounted for (Karanth and Nichols, 2002; MacKenzie et al., 2002, 2006). The cost, effort and complex logistics needed to overcome these problems frequently restrict the extent of carnivore

\footnotetext{
* Corresponding author at: University of Brighton, Biology Division, Huxley Building, Lewes Road, Moulsecoomb, Brighton BN2 4GJ, UK.

E-mail addresses: Thorn_Green@Hotmail.com (M. Thorn), Pwbateman@zoology. up.ac.za (P.W. Bateman), Stephen.waite@hartpury.ac.uk (S. Waite), Dawn.Scott@ Brighton.ac.uk (D.M. Scott).

1 Tel.: +44 (0)1273 642071; fax: +44 (0)1273 642674
}

surveys, so there are few examples of practical, unbiased protocols for use across large, heterogeneous areas.

One possible solution is the use of occupancy surveys: an increasingly popular method of assessing patterns and determinants of occurrence for a broad range of animal taxa. Examples include amphibians (MacKenzie et al., 2002; Mattfeldt et al., 2009; Sewell et al., 2010), birds (e.g. Watson et al., 2008) and mammals (Linkie et al., 2007; Thorn et al., 2009). Occupancy surveys have been implemented world-wide, at scales ranging from small habitat patches to the whole of India (Karanth et al., 2009). They require collection of detection/non-detection data, which can often be achieved with low cost and effort compared to demographic data (Thorn et al., 2010). Information theoretic modelling is typically used to correct for imperfect detection (false absence or presence) and temporal or spatial variations in detection probability (MacKenzie et al., 2002, 2006). This produces unbiased maximum likelihood estimates of numerous variables relevant to a wide variety of research, conservation and management applications (MacKenzie et al., 2006). Metric or categorical covariates can also be modelled to infer relationships between observed patterns 
and the underlying processes that cause them, and thereby project patterns in un-surveyed areas (MacKenzie et al., 2006).

Unfortunately, despite their versatility these methods are not without drawbacks. Occupancy modelling requires moderate to large sample sizes to achieve precise occupancy estimates (MacKenzie et al., 2002, 2006). Some form of replication at site level is also required to estimate detection probability (MacKenzie et al., 2002, 2006). The optimum number of sites and replicates depend on sampling strategy, as well as the occupancy and detectability of the focal species (Mackenzie and Royle, 2005). In some situations, $>50$ replicates per site may be necessary to achieve robust occupancy estimates with standard error $<0.05$ (Mackenzie and Royle, 2005). This can be achieved with multiple, independent observers or methods, but temporal replication is more common. It entails repeatedly surveying sites within an overall period short enough to minimise the likelihood of changes in occupancy during sampling (Hines et al., 2010; MacKenzie et al., 2002, 2006). Spatial replication is possible, but requires the same effort using independent replicates (e.g. transects), selected randomly, with replacement (Hines et al., 2010). However, newly-developed methods estimate detection probability from consecutive, spatially autocorrelated replicates (trail segments), that do not need to be randomly selected (see Hines et al., 2010). Only one visit to a site is required with no need for multiple independent spatial replicates, observers or methods. This may alleviate cost, effort and logistical limitations of replication by other means (Hines et al., 2010), especially in remote study areas, where difficult conditions hinder travel.

The Hines et al. (2010) method was developed for tigers (Panthera tigris), but should be readily transferrable to studies of other mobile species (Hines et al., 2010). We adapted it for use in a survey of brown hyaenas (Hyaena brunnea). The species is classified as near threatened, partly due to habitat loss and because although predominantly scavengers, they are frequently persecuted for suspected livestock predation (Wiesel et al., 2008). With estimated global population numbers $<10,000$ mature individuals, a $10 \%$ population decline over three generations would cause brown hyaenas to be re-classified as vulnerable (Wiesel et al., 2008). The species occurs within a restricted distribution range in the South West Arid Zone of Southern Africa (Wiesel et al., 2008). Hence, climate change also poses a potential threat. On-going assessment of remaining populations is therefore essential to informed management and conservation. Field surveys are needed to quantify abundance and distribution (Friedmann and Daly, 2004), monitor trends and identify causal factors (Mattfeldt et al., 2009). There have been no recent estimates of the number of individuals in South Africa. However, the latest conservation assessment indicates that numbers may be increasing where people are better educated about the species (presumably engendering more tolerant attitudes toward them), and where land use has shifted from intensive agriculture to wildlife farming (Friedmann and Daly, 2004).

The Hines et al. (2010) tiger protocol involves sign surveys (counts of scats, tracks, feeding signs, etc.) that should be generally suitable for species that leave visible signs of their presence when they use roads and trails. Such surveys have been successfully used to study a broad spectrum of mammals, including our focal species (Thorn et al., 2010). The tiger survey covered 22,000 $\mathrm{km}^{2}$ and population size was not estimated, although the authors suggest a technique for doing so. Our aim was to extend the 'tigers on trails' approach to a larger spatial scale and asses its utility for estimating African carnivore abundance and distribution. We employed a random sampling design with the objective of estimating brown hyaena occupancy and population size at landscape-scale. We also investigated our a priori hypothesis that attitude to carnivores and land area used for wildlife farming are important determinants of brown hyaena occupancy. We expected both factors to positively influence occupancy, but were unsure of the relative importance and magnitude of those effects. We analysed our data using new occupancy models that account for spatial auto-correlation (Hines et al., 2010). Finally, we used simulated data to assess the bias, precision and occupancy monitoring power of protocols based on the same survey design, but with differing levels of effort.

\section{Materials and methods}

\subsection{Study area}

The North West Province of South Africa covers $116,320 \mathrm{~km}^{2}$, is located between $25-28^{\circ} \mathrm{S}$ and $22-28^{\circ} \mathrm{E}$ and is bordered by Botswana to the north (Fig. 1). It is one of the most environmentally stressed areas of South Africa, where widespread land conversion and degradation (Hoffman and Ashwell, 2001) have caused deterioration and fragmentation of natural brown hyaena habitat. Most of the province is privately owned, approximately $10 \%$ is state-owned or tribal land and protected areas comprise just $2833 \mathrm{~km}^{2}$ (Tladi et al., 2002). $62,979 \mathrm{~km}^{2}$ of the province is used for crop production, irrigation, domestic livestock or wildlife farming (mainly non-domesticated ungulates often referred to as game). Our surveys focussed on these extensive, heterogeneous agricultural areas, where brown hyaenas and several other medium and large-sized carnivore species co-exist with people.

\subsection{Sampling design and field methods}

The largest home range for resident brown hyaenas in an area of Botswana that is ecologically similar to the North West Province was $221 \mathrm{~km}^{2}$ (Maude, 2005). However, $\approx 8 \%$ of individuals are nomadic (Mills, 1982) and in the Botswana study the largest home range for a non-resident male was $505 \mathrm{~km}^{2}$ (Maude, 2005). This equates to a range diameter of $22.47 \mathrm{~km}$ so we set our grid-based sampling frame to the nearest interval of the decimal degree coordinate system ( 1 grid cell $=20.39 \mathrm{~km} \times 20.39 \mathrm{~km}$ ). Grid cell dimensions therefore slightly exceeded expected maximum home range size (Hines et al., 2010) of $\geqslant 92 \%$ of brown hyaenas.

We overlaid our grid on the study area using ArcView GIS 3.3 and excluded protected areas and grid cells used solely for nonagricultural purposes. We then randomly selected 25 grid cells, henceforth termed 'sites'. The total area of the 25 survey sites was $10,390 \mathrm{~km}^{2}$, equating to $16.9 \%$ of agricultural land in the province. Selection criteria included a separation of $\geqslant 1$ grid cell to minimise the likelihood of individuals moving between sites during sampling. For the same reason, sites close to one another were surveyed successively. Survey duration was limited to 5 months to minimise the likelihood of changes in brown hyaena distribution and occupancy during sampling (Hines et al., 2010).

Sign surveys followed roads within each site chosen to maximise detection probability, thereby minimising the likelihood of 'false absences' (MacKenzie et al., 2006). Hines et al. (2010) divided survey routes into spatially auto-correlated replicates consisting of $1 \mathrm{~km}$ trail segments. Thorn et al. (2010) gave naive occupancy estimates of 0.64 from sign surveys. We assumed moderate detection probability (0.4-0.6) because brown hyaena sign is conspicuous and easily differentiated from that of other carnivores inhabiting the study area. Applying those values to Mackenzie and Royle's (2005) findings, we determined that $251 \mathrm{~km}$ segments per site should provide sufficient detections for precise occupancy estimates, even if it were necessary to amalgamate segments during analysis. Following Hines et al. (2010), the number of segments surveyed at each site was proportionate to the area of agricultural land it contained, resulting in actual effort of $11 \mathrm{~km}$ to $25 \mathrm{~km}$. If this sampling effort could not be achieved in one property, we 
(a)
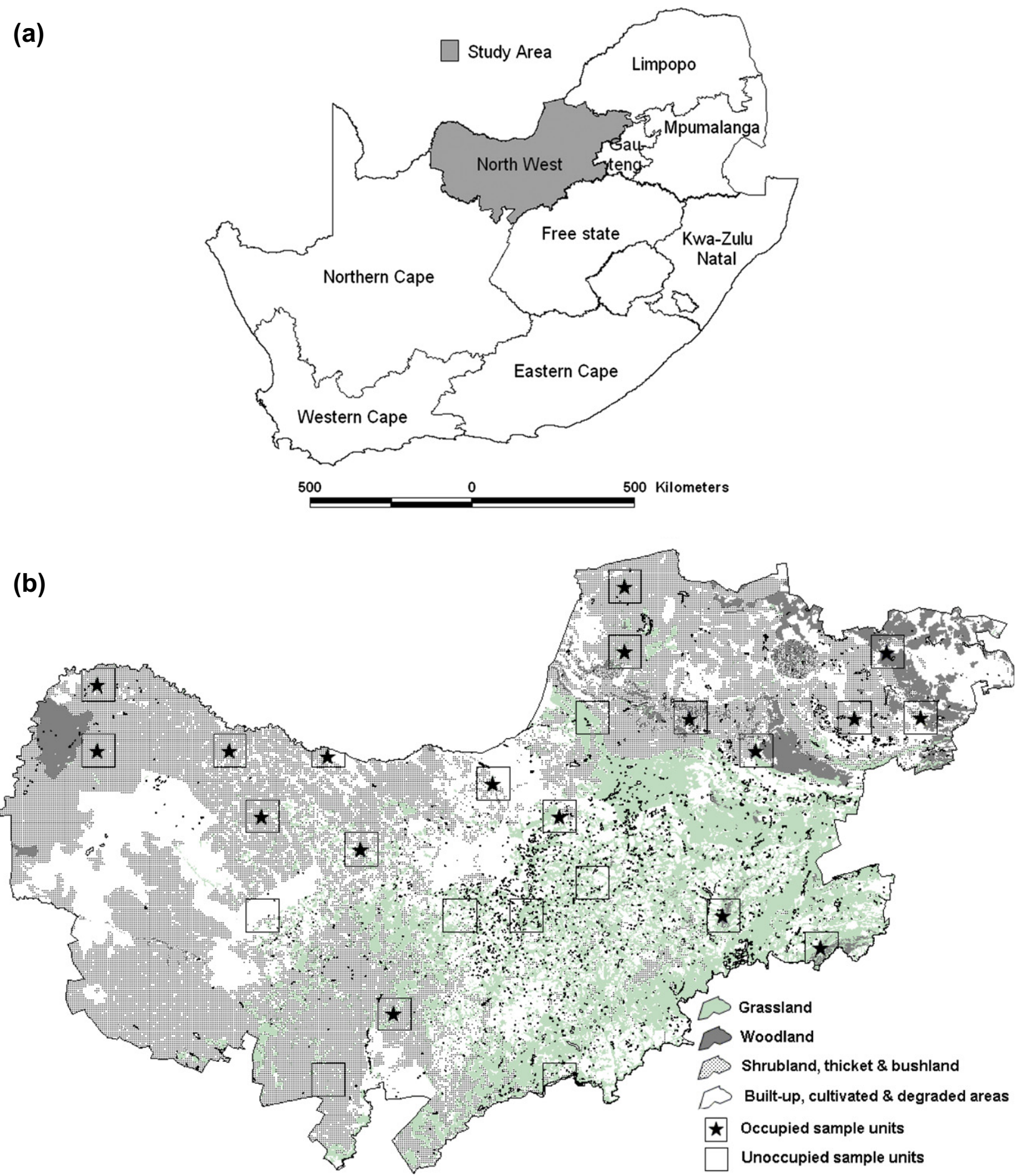

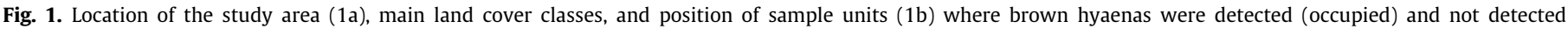
(unoccupied).

surveyed another property within the site and pooled the data. On rare occasions when land owners in the randomly selected site were un-contactable or unwilling to allow access, we surveyed the closest alternative site.

We surveyed during the dry season, when sign persists longest because of decreased weathering and coprophage abundance. Two experienced observers conducted all surveys from a vehicle travelling $10-15 \mathrm{~km} / \mathrm{h}$, using the odometer to measure consecutive $1 \mathrm{~km}$ replicates. We searched for brown hyaena sign on and within a $2 \mathrm{~m}$ strip either side of un-tarred farm roads (Thorn et al., 2010). The primary observer was seated in a spotter's chair fixed above the front bumper and the secondary observer looked for sign from within the vehicle. We identified sign to species based on colour, dimensions, position and presence of accompanying signs (Stuart and Stuart, 2000).

We collected site-specific covariate data during standardised, semi-structured interviews with the manager or owner of each property where we completed sign surveys (i.e. up to two 
interviews per site). Respondents estimated the land area $\left(\mathrm{km}^{2}\right)$ within their property used for wildlife farming. We assessed attitudes to carnivores using nine statements (e.g. 'this site cannot tolerate any predators' and 'it does not matter if predators kill a few of my animals'), which respondents rated on a five point Likert scale (Zimmermann et al., 2005). Answers were coded from one (strongly negative) to five (strongly positive) and summed to give an attitude score per respondent. We tested internal consistency using Chronbach's alpha (0.78), confirming that the summed score was truly additive and reflected overall attitude (Zimmermann et al., 2005). If two properties were surveyed within the site, mean covariate values were used in occupancy modelling. We also included a survey-specific covariate for substrate quality to minimise un-modelled sources of heterogeneity (MacKenzie et al., 2006). Replicates with substrate that favoured detection of sign (e.g. fine, shallow soil) were coded ' 1 ' and those where scat and particularly tracks were hard to detect (e.g. deep sand or rocky areas) were coded ' 0 '.

\subsection{Analysis}

We constructed standard detection histories for each site (MacKenzie et al., 2006) and converted site-specific covariates $\left(\mathrm{km}^{2}\right.$ used for wildlife farming and summed attitude score) to standard normalised scores (Donovan and Hines, 2007). We then imported detection histories and corresponding covariate values into PRESENCE v.3.0 (Hines, 2006).

The new spatial auto-correlation models estimate four parameters, even without covariates (Hines et al., 2010), and in view of our sample size, we considered it likely that models containing covariates might fail to converge. We overcame this problem using the approach recommended by Hines et al. (2010) for abundance estimation. We fitted the spatial auto-correlation model without covariates and used the results to determine the cumulative segment length at which detection of the focal species ceased to be dependent on detection in the preceding segment. At that length, we would expect $\theta \approx \theta^{\prime}$, where $\theta=$ probability of the species being present at a replicate (i.e. segment) given the previous replicate (i.e. segment) was not occupied by the species, and $\theta^{\prime}=$ probability of the species being present at a replicate (i.e. segment) given the previous replicate (i.e. segment) was occupied by the species. Detection histories were collapsed to that segment length, generating approximately independent replicates suitable for use with standard PRESENCE models. We aggregated substrate quality values using the mode of values in the collapsed segments.

We began by fitting candidate single-season models, where occupancy $(\psi)$ was first assumed constant, and then allowed to vary with individual or additively combined site-specific covariates. For each permutation of $\psi$, detection probability $(\rho)$ was first assumed constant and then held in a general model (see MacKenzie et al., 2006, pp. 113-116). We ranked candidate models in order of parsimony using Akaike Information Criterion (AIC) (Burnham and Anderson, 2002). The small sample correction AICc was not used because effective sample size could not be identified (Hines et al., 2010; MacKenzie et al., 2006). We used Akaike model weight to determine relative evidence in favour of each model, and summed weights to determine the relative influence of site-specific covariates (Burnham and Anderson, 2002; MacKenzie et al., 2006). We model averaged maximum likelihood parameter estimates ( $\psi$ and $\hat{\rho}$ ) from all models with $>0.01$ Akaike weight (Linkie et al., 2007). Parameter estimates from models with site or survey-specific parameter values, were calculated as an average of the values, weighted by the number of sites or surveys in which they occurred (MacKenzie et al., 2006). The fit of the global model was assessed using the parametric bootstrap procedure implemented in PRESENCE (MacKenzie and Bailey, 2004).
We used the same modelling approach to estimate brown hyaena population size from the Royle-Nichols abundance induced heterogeneity model (henceforth the Royle-Nichols model) in PRESENCE. Like the single-season model, this model also assumes data from independent survey replicates, so we again used the collapsed detection history and covariates. However, it was not possible to assess goodness of fit for the Royle-Nichols or spatial auto-correlation models. Goodness of fit statistics are not currently reported by PRESENCE and further research is needed to develop appropriate tests (Linkie et al., 2010).

The Royle-Nichols model provides estimates of the parameters $\lambda$ and $r$, representing average abundance per site and innate species detectability respectively (Royle and Nichols, 2003). The parameter $\lambda$ can be interpreted as an index of abundance. However, if detection of individuals is independent and site-specific abundance of individuals follows a Poisson distribution (which is the mixture distribution used in PRESENCE models), $\lambda$ may also be interpreted as the expected number of individuals per sample unit (MacKenzie et al., 2006). We took this approach and divided $\lambda$ by the sampling unit area $\left(415.75 \mathrm{~km}^{2}\right)$ to estimate average brown hyaena density in the sites we surveyed. Our sampling design made it unlikely that the same individuals were detected in two survey sites, but we considered it likely that individuals detected in survey sites might also use adjacent grid cells. Thus, overall density would depend on the number of grid cells used by individuals (J. Nichols, personal communication). We therefore divided $\lambda$ by the average number of grid cells used by brown hyaenas, to estimate overall density across surveyed and un-surveyed sites.

To determine the optimum combination of site and replicate numbers, we used our parameter estimates as 'true' values and simulated data under various sampling permutations (1000 iterations each). We capped effort at 75 sites (50\% of total sites in agricultural land) and 12 segments ( $48 \mathrm{~km}$ ) per site because survey designs requiring greater effort would increasingly counteract methodological benefits. For each sampling permutation we approximated bias $(E[\hat{\phi}-\phi])$, relative bias (bias $[\hat{\phi}] / \phi)$, coefficient of variation $(\mathrm{CV}=\mathrm{SE}(\hat{\phi}) / \hat{\phi})$, and root mean square error $\left(\right.$ RMSE $\left.=\sqrt{(E[\hat{\phi}]-\phi)^{2}}\right)$, where $\phi$, and $\hat{\phi}$ denote the true parameter value used to generate the data and the simulated estimator respectively, and $E \hat{\phi}$ and $C V$ are calculated from the mean of 1000 estimates in each simulated data set (Bailey et al., 2007). RMSE incorporates the sum of variance and squared bias, thus offering a combined measure of bias and precision (Bailey et al., 2007).

We investigated how sample size (number of sites) affected statistical power to detect $30 \%$ higher, or $30 \%$ and $50 \%$ lower occupancy, under Type I (false positive) error probabilities $(\alpha)$ of 0.05 , 0.1 and 0.2 . Power is defined as $1-\beta$, where $\beta$ is the probability of Type II error (false negative). We selected $30 \%$ as an effect size that was probably large enough to be detectable with realistic sample sizes, but small enough to be reversible through management intervention (Barlow et al., 2008). We modelled the 50\% decline largely to explore the trade off between effect size and sample size. To generate occupancy declines, we used multi-season models and numeric analytic large-sample approximations (Bailey et al., 2007). Initial occupancy was 0.748 , detection probability was constant at 0.477 for all models, and for simplicity, two replicates per site were assumed. As we are not aware of relevant estimates from carnivore survey data we chose arbitrary values of extinction and colonisation probability to generate changes in occupancy. These values reflect hypothetical scenarios in which the probability that sites will become unoccupied (extinction) and the probability that unoccupied sites will be re-colonised produces a net gain or loss in the number of sites occupied. Extinction probabilities of $0.02,0.19$ and 0.33 and corresponding colonisation probabilities of $0.7,0.18$ 
and 0.17 produced $+30 \%,-30 \%$ and $-50 \%$ changes respectively. We used a likelihood ratio test (LRT) to compare each change model (the alternative hypothesis, $H_{a}$ ) with a nested model that assumed no change in occupancy over time (the null model, $H_{o}$ ) (Mattfeldt et al., 2009). The Royle-Nichols model has not been extended to multi-season estimation, so it was not possible to use a similar approach for abundance.

Simulated data and numeric-analytic approximations were generated using the program GENPRES (Bailey et al., 2007) and LRT tests were conducted in program MARK (White and Burnham, 1999).

\section{Results}

We completed $577 \mathrm{~km}$ of sign surveys between 13th May and 10th October 2008 and detected brown hyaena sign in 85 segments at 18 of the 25 sites, giving a naive occupancy estimate of 0.72 .

Using $1 \mathrm{~km}$ segments, the spatial auto-correlation model $\psi($.$) ,$ $\theta(),. \theta^{\prime}(),. \rho($.$) provided estimates of \hat{\theta}=0.175$ ( \pm SE 0.022$)$ and $\hat{\theta^{\prime}}=0.333( \pm$ SE 0.052$)$, indicating strong between-segment dependence. We collapsed brown hyaena detection histories to $4 \mathrm{~km} \mathrm{seg-}$ ments because this came closest to $\theta \approx \theta^{\prime}$, producing estimates of $\hat{\theta}=0.447( \pm$ SE 0.070$)$ and $\hat{\theta}^{\prime}=0.529( \pm$ SE 0.070). Hines et al. (2010) found that standard models considerably under-estimated occupancy when spatial auto-correlation was not properly accounted for. We compared the occupancy estimate from our top ranked single-season model, based on $4 \mathrm{~km}$ segments (Table 1 ), with single season and spatial auto-correlation models, based on $1 \mathrm{~km}$ segments. As expected, the $1 \mathrm{~km}$ standard single-season model produced a lower estimate $(\hat{\psi}=0.733)$. The $1 \mathrm{~km}$ spatial auto-correlation model produced an estimate matching our result $(\hat{\psi}=0.761)$, confirming that $4 \mathrm{~km}$ segments provided a reasonable approximation of independence. Hence, although our results were generated from standard PRESENCE models, we would expect similar results from spatial-autocorrelation models.

There was no evidence of a lack of fit in the global $4 \mathrm{~km}$ segment single-season model (average $\chi^{2}=11.49, P=0.30$ ) and we did not adjust standard errors as there was no evidence of over-dispersion $(\hat{c}=1.05)$. With $\leqslant 14 \%$ coefficient of variation $(\mathrm{CV})$, precision of both

Table 1

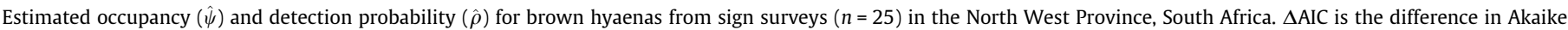

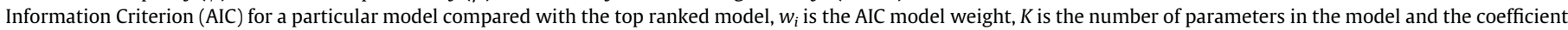

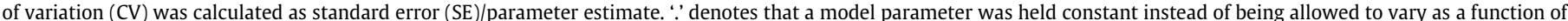
the covariates landowner attitude to carnivores, land area used for wildlife farming and substrate suitability for sign surveys.

\begin{tabular}{|c|c|c|c|c|c|c|c|}
\hline Model & $\Delta \mathrm{AIC}$ & $w_{i}$ & $K$ & $\hat{\psi}( \pm \mathrm{SE})$ & $\mathrm{CV} \hat{\psi}$ & $\hat{\rho}( \pm \mathrm{SE})$ & $\mathrm{CV} \hat{\rho}$ \\
\hline$\psi$ (attitude $+\mathrm{km}^{2}$ wildlife), $p$ (substrate) & 0.00 & 0.452 & 5 & $0.761(0.093)$ & 0.12 & $0.477(0.063)$ & 0.13 \\
\hline$\psi($ attitude), $p$ (substrate) & 1.24 & 0.243 & 4 & $0.761(0.093)$ & 0.12 & $0.477(0.064)$ & 0.13 \\
\hline$\psi\left(\mathrm{km}^{2}\right.$ wildlife $), p$ (substrate) & 2.38 & 0.137 & 4 & $0.729(0.105)$ & 0.14 & $0.491(0.066)$ & 0.14 \\
\hline$\psi\left(\right.$ attitude $+\mathrm{km}^{2}$ wildlife), $p()$. & 3.98 & 0.067 & 4 & $0.735(0.133)$ & 0.18 & $0.486(0.051)$ & 0.11 \\
\hline$\psi\left(\mathrm{km}^{2}\right.$ wildlife $), p()$. & 4.49 & 0.048 & 3 & $0.727(0.105)$ & 0.15 & $0.491(0.048)$ & 0.10 \\
\hline$\psi($ attitude), $p()$. & 5.12 & 0.035 & 3 & $0.750(0.155)$ & 0.21 & $0.476(0.055)$ & 0.11 \\
\hline$\psi(),$.$p (substrate)$ & 6.51 & 0.017 & 3 & $0.733(0.092)$ & 0.13 & $0.488(0.067)$ & 0.14 \\
\hline$\psi(),. p()$. & 8.53 & 0.006 & 2 & $0.732(0.092)$ & 0.13 & $0.488(0.049)$ & 0.10 \\
\hline Model Averaged Estimates & & & & $0.748(0.100)$ & 0.13 & $0.477(0.062)$ & 0.13 \\
\hline
\end{tabular}

Table 2

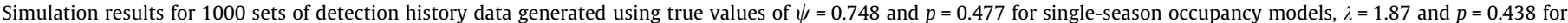

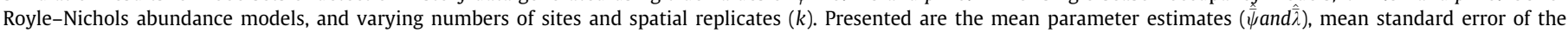

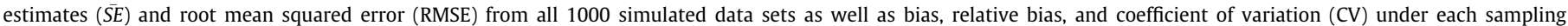
permutation.

\begin{tabular}{|c|c|c|c|c|c|c|c|c|c|c|c|c|c|}
\hline Sites & $k$ & $\hat{\bar{\psi}}$ & $\overline{S E} \hat{\psi}$ & Bias $\hat{\bar{\psi}}$ & Relative bias $\hat{\bar{\psi}}$ & $\mathrm{CV} \hat{\bar{\psi}}$ & RMSE $\hat{\psi}$ & $\hat{\bar{\lambda}}$ & $\overrightarrow{S E} \hat{\lambda}$ & Bias $\hat{\bar{\lambda}}$ & Relative bias $\hat{\bar{\lambda}}$ & $\mathrm{CV} \hat{\bar{\lambda}}$ & RMSE $\hat{\lambda}$ \\
\hline 25 & 3 & 0.784 & 0.127 & $3.6 \%$ & $1.9 \%$ & $16.2 \%$ & 0.128 & 1.970 & 0.668 & $10.0 \%$ & $5.4 \%$ & $33.9 \%$ & 0.673 \\
\hline 25 & 6 & 0.761 & 0.088 & $1.3 \%$ & $0.7 \%$ & $11.6 \%$ & 0.095 & 1.874 & 0.355 & $0.4 \%$ & $0.2 \%$ & $19.0 \%$ & 0.226 \\
\hline 25 & 9 & 0.758 & 0.084 & $1.0 \%$ & $0.6 \%$ & $11.1 \%$ & 0.084 & 1.858 & 0.312 & $-1.2 \%$ & $-0.6 \%$ & $16.8 \%$ & 0.148 \\
\hline 25 & 12 & 0.757 & 0.084 & $0.9 \%$ & $0.5 \%$ & $11.0 \%$ & 0.083 & 1.852 & 0.294 & $-1.8 \%$ & $-0.9 \%$ & $15.9 \%$ & 0.112 \\
\hline 35 & 3 & 0.763 & 0.111 & $1.5 \%$ & $0.8 \%$ & $14.6 \%$ & 0.112 & 1.965 & 0.521 & $9.5 \%$ & $5.1 \%$ & $26.5 \%$ & 0.502 \\
\hline 35 & 6 & 0.760 & 0.075 & $1.2 \%$ & $0.6 \%$ & $9.8 \%$ & 0.076 & 1.888 & 0.300 & $1.8 \%$ & $1.0 \%$ & $15.9 \%$ & 0.184 \\
\hline 35 & 9 & 0.750 & 0.072 & $0.2 \%$ & $0.1 \%$ & $9.6 \%$ & 0.072 & 1.877 & 0.264 & $0.7 \%$ & $0.4 \%$ & $14.1 \%$ & 0.121 \\
\hline 35 & 12 & 0.754 & 0.071 & $0.6 \%$ & $0.3 \%$ & $9.5 \%$ & 0.072 & 1.859 & 0.250 & $-1.1 \%$ & $-0.6 \%$ & $13.5 \%$ & 0.101 \\
\hline 45 & 3 & 0.765 & 0.099 & $1.7 \%$ & $0.9 \%$ & $12.9 \%$ & 0.099 & 1.947 & 0.422 & $7.7 \%$ & $4.1 \%$ & $21.7 \%$ & 0.369 \\
\hline 45 & 6 & 0.753 & 0.067 & $0.5 \%$ & $0.3 \%$ & $8.9 \%$ & 0.068 & 1.892 & 0.263 & $2.2 \%$ & $1.2 \%$ & $13.9 \%$ & 0.165 \\
\hline 45 & 9 & 0.753 & 0.064 & $0.5 \%$ & $0.2 \%$ & $8.5 \%$ & 0.067 & 1.872 & 0.232 & $0.2 \%$ & $0.1 \%$ & $12.4 \%$ & 0.107 \\
\hline 45 & 12 & 0.754 & 0.063 & $0.6 \%$ & $0.3 \%$ & $8.4 \%$ & 0.064 & 1.868 & 0.220 & $-0.2 \%$ & $-0.1 \%$ & $11.8 \%$ & 0.088 \\
\hline 55 & 3 & 0.755 & 0.089 & $0.7 \%$ & $0.4 \%$ & $11.8 \%$ & 0.093 & 1.916 & 0.367 & $4.6 \%$ & $2.5 \%$ & $19.2 \%$ & 0.317 \\
\hline 55 & 6 & 0.754 & 0.060 & $0.6 \%$ & $0.3 \%$ & $8.0 \%$ & 0.061 & 1.876 & 0.235 & $0.6 \%$ & $0.3 \%$ & $12.5 \%$ & 0.147 \\
\hline 55 & 9 & 0.750 & 0.058 & $0.2 \%$ & $0.1 \%$ & $7.7 \%$ & 0.062 & 1.860 & 0.208 & $-1.0 \%$ & $-0.5 \%$ & $11.2 \%$ & 0.097 \\
\hline 55 & 12 & 0.753 & 0.058 & $0.5 \%$ & $0.2 \%$ & $7.6 \%$ & 0.062 & 1.863 & 0.199 & $-0.7 \%$ & $-0.4 \%$ & $10.7 \%$ & 0.073 \\
\hline 65 & 3 & 0.765 & 0.082 & $1.7 \%$ & $0.9 \%$ & $10.7 \%$ & 0.086 & 1.915 & 0.336 & $4.5 \%$ & $2.4 \%$ & $17.5 \%$ & 0.291 \\
\hline 65 & 6 & 0.752 & 0.056 & $0.4 \%$ & $0.2 \%$ & $7.4 \%$ & 0.055 & 1.882 & 0.216 & $1.2 \%$ & $0.7 \%$ & $11.5 \%$ & 0.129 \\
\hline 65 & 9 & 0.754 & 0.053 & $0.6 \%$ & $0.3 \%$ & $7.1 \%$ & 0.056 & 1.875 & 0.193 & $0.5 \%$ & $0.3 \%$ & $10.3 \%$ & 0.090 \\
\hline 65 & 12 & 0.753 & 0.053 & $0.5 \%$ & $0.3 \%$ & $7.0 \%$ & 0.052 & 1.867 & 0.183 & $-0.3 \%$ & $-0.2 \%$ & $9.8 \%$ & 0.064 \\
\hline 75 & 3 & 0.761 & 0.077 & $1.3 \%$ & $0.7 \%$ & $10.1 \%$ & 0.077 & 1.919 & 0.308 & $4.9 \%$ & $2.6 \%$ & $16.1 \%$ & 0.273 \\
\hline 75 & 6 & 0.753 & 0.052 & $0.5 \%$ & $0.2 \%$ & $6.9 \%$ & 0.052 & 1.879 & 0.200 & $0.9 \%$ & $0.5 \%$ & $10.6 \%$ & 0.118 \\
\hline 75 & 9 & 0.751 & 0.050 & $0.3 \%$ & $0.1 \%$ & $6.6 \%$ & 0.050 & 1.870 & 0.178 & $0.0 \%$ & $0.0 \%$ & $9.5 \%$ & 0.081 \\
\hline 75 & 12 & 0.752 & 0.049 & $0.4 \%$ & $0.2 \%$ & $6.6 \%$ & 0.050 & 1.871 & 0.170 & $0.1 \%$ & $0.1 \%$ & $9.1 \%$ & 0.063 \\
\hline
\end{tabular}


$\hat{\psi}$ and $\hat{\rho}$ was good (Table 1 ). The model averaged estimate of brown hyaena occupancy was slightly higher than the naive estimate, so we may have failed to detect the species in one site where it was present (Table 1). Summed Akaike weights for site covariates were similar with 0.79 for attitude score $(\beta=2.663 \pm$ SE 1.718$)$ and 0.70 for wildlife farm area $(\beta=8.628 \pm$ SE 6.587$)$, indicating that both have a strong positive influence on occupancy. In the top ranked model, sites with above average attitude scores had $89 \%$ higher mean estimated occupancy than sites where attitude was below average $(\hat{\bar{\psi}}=0.991 \pm \mathrm{SE} \quad 0.019$ compared with $\hat{\bar{\psi}}=0.523 \pm \mathrm{SE}$ 0.160 ). It seems that presence of wildlife farms is more important than their land area, although area exerts a lesser positive influence on occupancy. Mean estimated occupancy was 57\% higher in sites that had wildlife farms than in sites that had none ( $\hat{\bar{\psi}}=0.906 \pm$ SE 0.057 compared with $\hat{\bar{\psi}}=0.577 \pm$ SE 0.138 ). Of the sites that did have wildlife farms, those with $\geqslant$ average area used for wildlife farming had 15\% higher mean estimated occupancy than those with $<$ average wildlife farming area $(\hat{\bar{\psi}}=0.979 \pm \mathrm{SE}$ 0.052 compared with $\hat{\bar{\psi}}=0.851 \pm$ SE 0.060 ). The odds of finding brown hyaena sign in segments with good substrate were 2.5 times higher than in segments with poor substrate $(\beta=0.924 \pm$ SE0.378).
The $\triangle$ AIC between the top and bottom ranked Royle-Nichols abundance induced heterogeneity models was 1.04 and all eight candidate models achieved similar Akaike weight, indicating that all were equally well supported. It seems our sample size was insufficient to differentiate covariate effects in this model (Royle and Nichols, 2003). We therefore took our parameter estimates from the constant model $\hat{\lambda}(),. \hat{r}($.) This gave $\hat{\lambda}=1.867$ ( \pm SE 0.507 , $\mathrm{CV}=27 \%), \hat{r}=0.438( \pm \mathrm{SE} 0.088, \mathrm{CV}=20 \%)$, and average expected density in our 25 grid cells of $0.45 / 100 \mathrm{~km}^{2}$ (i.e. $\hat{\lambda} / 4.16 \mathrm{~km}^{2}$ ). When we overlaid our sampling frame on home range figures in Maude (2005) the mean number of grid cells used by individual brown hyaenas was 3 . This gives an overall density estimate across surveyed and un-surveyed grid cells of $0.45 / 3=0.15 / 100 \mathrm{~km}^{2}(95 \%$ confidence interval, $0.07-0.23 / 100 \mathrm{~km}^{2}$ ).

Low RMSE indicates low bias and high precision. Unsurprisingly, simulations (Table 2) showed that greater effort is required to obtain low RMSE for abundance estimates compared with occupancy estimates. The largest decrease in RMSE of both parameters occurred when effort increased from three to six replicates per site (Fig. 2). Further increases in the number of replicates conferred diminishing incremental benefit. However, at effort of six replicates per site, RMSE of abundance estimates was up to $139 \%$ greater than

(a) Occupancy

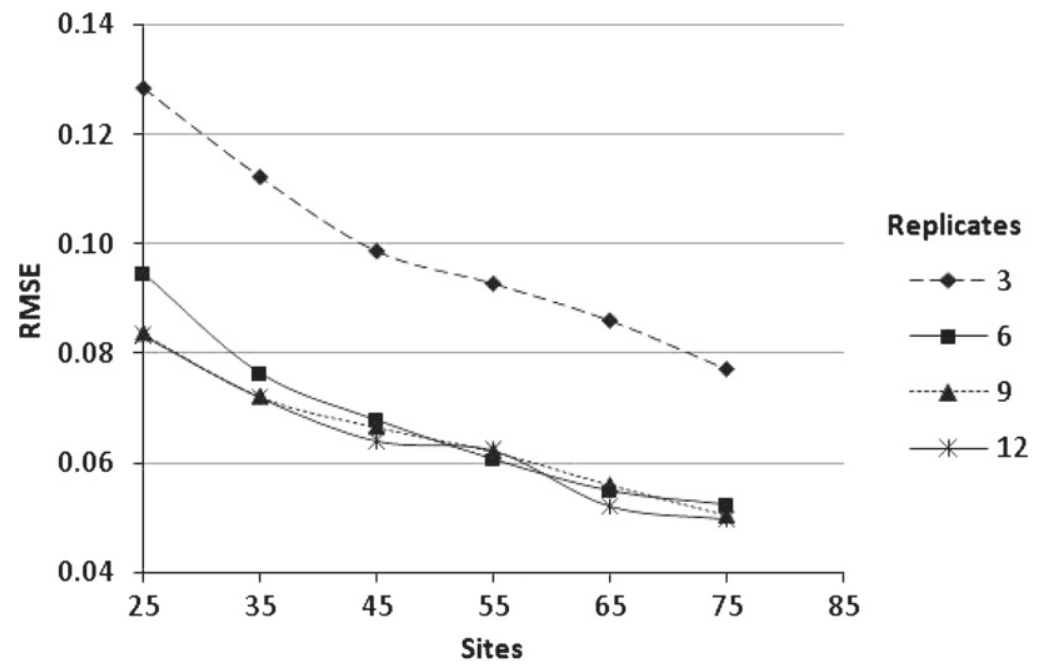

(b) Abundance

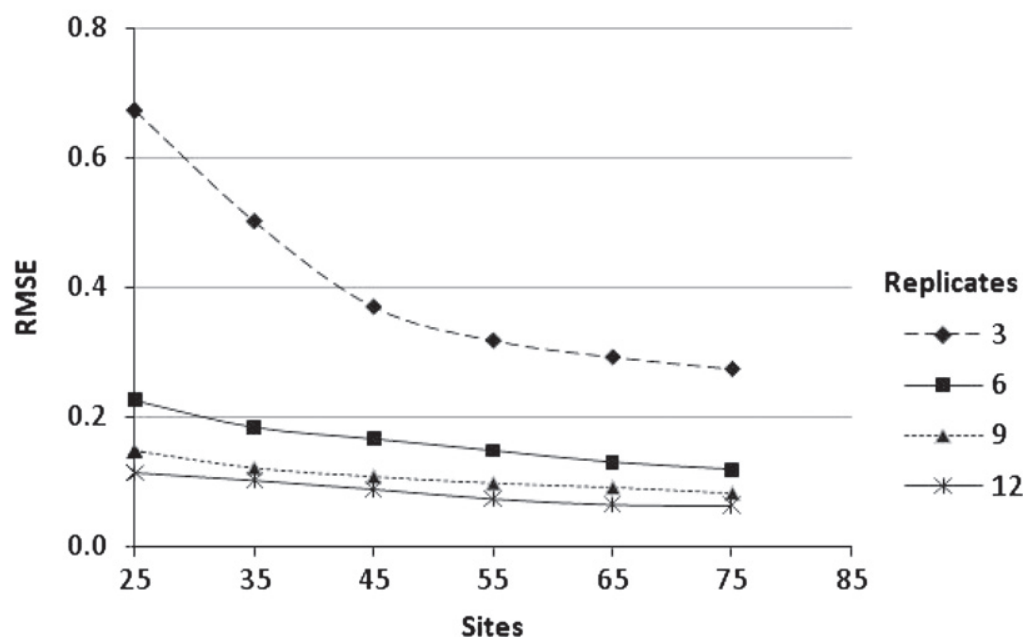

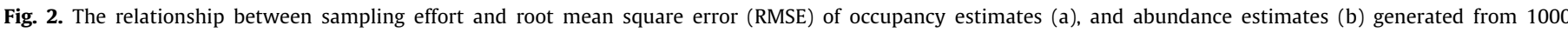
simulations of each sampling permutation (Table 2). 
Table 3

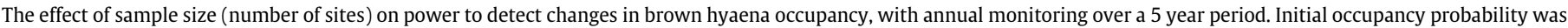

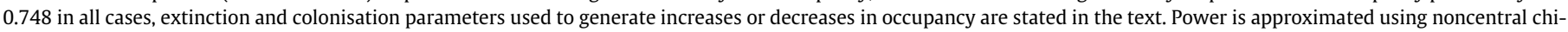
square statistics from likelihood ratio tests (Burnham et al., 1987).

\begin{tabular}{|c|c|c|c|c|c|c|c|c|c|c|c|c|}
\hline \multirow[b]{2}{*}{ Sites } & \multicolumn{4}{|c|}{$30 \%$ increase: } & \multicolumn{4}{|c|}{$30 \%$ decrease: } & \multicolumn{4}{|c|}{ 50\% decrease: } \\
\hline & $X_{4}^{2}$ & Power $\alpha 0.05$ & Power $\alpha 0.1$ & Power $\alpha 0.2$ & $X_{4}^{2}$ & Power $\alpha 0.05$ & Power $\alpha 0.1$ & Power $\alpha 0.2$ & $X_{4}^{2}$ & Power $\alpha 0.05$ & Power $\alpha 0.1$ & Power $\alpha 0.2$ \\
\hline 25 & 0.81 & $9 \%$ & $17 \%$ & $29 \%$ & 1.31 & $13 \%$ & $21 \%$ & $34 \%$ & 3.79 & $30 \%$ & $43 \%$ & $58 \%$ \\
\hline 35 & 1.14 & $11 \%$ & $19 \%$ & $33 \%$ & 1.84 & $16 \%$ & $26 \%$ & $40 \%$ & 5.30 & $42 \%$ & $55 \%$ & $69 \%$ \\
\hline 45 & 1.46 & $13 \%$ & $22 \%$ & $36 \%$ & 2.36 & $20 \%$ & $30 \%$ & $45 \%$ & 6.81 & $53 \%$ & $65 \%$ & $78 \%$ \\
\hline 55 & 1.79 & $16 \%$ & $25 \%$ & $39 \%$ & 2.89 & $24 \%$ & $35 \%$ & $50 \%$ & 8.33 & $62 \%$ & $74 \%$ & $85 \%$ \\
\hline 65 & 2.11 & $18 \%$ & $28 \%$ & $43 \%$ & 3.41 & $28 \%$ & $39 \%$ & $55 \%$ & 9.84 & $71 \%$ & $81 \%$ & $89 \%$ \\
\hline 75 & 2.43 & $20 \%$ & $31 \%$ & $46 \%$ & 3.94 & $32 \%$ & $44 \%$ & $59 \%$ & 11.36 & $78 \%$ & $86 \%$ & $93 \%$ \\
\hline
\end{tabular}

RMSE of occupancy estimates (Table 2). At effort of 12 replicates per site, the difference was between $16 \%$ and $40 \%$ so this level of effort may be preferable in surveys attempting to estimate both parameters. In addition, although relative bias of occupancy and abundance estimates was not always optimal at 12 replicates, CV and RMSE were. Generally, percent decrease (improvement) in RMSE declined at sample sizes of $\geqslant 55$ sites for occupancy and $\geqslant 65$ for abundance, suggesting that lower sample sizes amplify bias and error.

Simulations with 25 sites and six replicates show much better precision than was achieved with equivalent effort in our field survey. This is probably due to missing observations in our survey (induced by scaled effort), which adversely affect precision (MacKenzie et al., 2002). As an example, the standard error estimate from our simulation would reduce the width of the $95 \%$ confidence interval for our overall density estimate by $30 \%$.

Power analysis (Table 3 ) showed that none of the sampling permutations we considered are likely to provide sufficient sensitivity to detect a $30 \%$ change in occupancy with $\geqslant 80 \%$ power. Even at $\alpha=0.2,145$ sites were required to detect a 30\% decline and 225 to detect an equivalent increase. By comparison, $\approx 80 \%$ power to detect a $50 \%$ decline in occupancy could be achieved with sample sizes $\geqslant 45$ sites, although 75 sites would be required to achieve that level of power with $\alpha=0.05$.

\section{Discussion}

Monitoring programmes are often criticised for a lack of scientific rigour, arising out of poor design or insufficiently robust statistical analysis (Linkie et al., 2010; Mattfeldt et al., 2009). We evaluated the efficacy of a new method that addresses these issues in a logistically efficient manner. Our assessment consisted of a field survey based on a probabilistic sampling design, permitting generalisation of the results. We then used simulations to extrapolate a protocol suitable for monitoring landscape-scale population trends. Our findings account for sources of bias (spatial variation, imperfect detection and spatial-autocorrelation) that give rise to unreliable inferences if overlooked. They also combine questionnaire and biophysical data in an occupancy modelling framework. We are aware of only two prior studies that have used this approach (Karanth et al., 2009; Zeller et al., 2011), which proved an efficient means of integrating social and biological factors that affect brown hyaena conservation status.

We accounted for spatial auto-correlation by aggregating transects to $4 \mathrm{~km}$, and considered the resulting estimates of $\hat{\theta}$ and $\hat{\theta}^{\prime}$ sufficiently close to conclude that there was no evidence of strong between-segment dependence. Overlapping confidence intervals support this assumption. However, a 0.08 discrepancy between $\hat{\theta}$ and $\hat{\theta}^{\prime}$ indicates some remaining between-segment dependence and it would be useful to quantify the effect of this residual auto-correlation. Achieving this would require larger sample sizes to enable a comparison of results from spatial-autocorrelation models with those from aggregated data used in standard singleseason models.

We also made a number of assumptions when interpreting the results from the Royle-Nichols model. We assume that the model fitted the data well, detection of individuals was independent, individuals were equally detectable across the whole sampling site, and site-specific abundance of individuals followed a Poisson distribution. We also presume that individuals in our study area use an average of three grid cells and have similar home range sizes to individuals in Botswana. Although these assumptions are not inherently unrealistic, they are untested, so our density estimate should be considered speculative at this point. Nevertheless, it seems reasonable to infer that brown hyaena density in our agricultural areas was at least an order of magnitude lower than density in or adjoining many protected areas. Examples of density estimates from protected areas include $1.8 / 100 \mathrm{~km}^{2}$ in the southern Kalahari (Mills, 1982), $\leqslant 2.0 / 100 \mathrm{~km}^{2}$ in Makgadikgadi, Botswana (Maude, 2005), and $2.8 / 100 \mathrm{~km}^{2}$ in the North West Province, South Africa (Thorn et al., 2009).

A number of anthropogenic and natural factors probably influence this disparity, but we focussed on two anthropogenic determinants that local experts consider particularly influential. It is not clear by what mechanism they benefit brown hyaenas. We assume that positive attitudes reduce direct persecution and unintentional brown hyaena mortality caused by lethal measures targeted at other carnivores, thus reducing extinction rates. Converting from intensive agricultural practices to wildlife farming allows natural succession of vegetation (Hejcmanova et al., 2010), increasing the frequency of good quality habitat patches (and possibly natural prey availability) in an environmentally stressed landscape. In addition, carnivores dispersing through a hostile human-modified matrix typically experience high mortality rates (Woodroffe and Ginsberg, 1998), and wildlife farms may therefore provide an important refuge from anthropogenic threats. Further surveys will be required to test these hypotheses and identify which additional factors exert the strongest beneficial or threatening influences. Such data would greatly assist optimal deployment of conservation effort and resources.

In the meantime, activities like education and outreach initiatives that promote positive attitudes are likely to increase the area occupied by brown hyaenas and may alleviate the threat of population declines due to persecution. Activities that encourage wildlife farming are likely to increase connectivity between subpopulations, facilitating gene flow and rescue effects in the event of patch level extinction. Thus, increased prevalence of positive attitudes and wildlife farming should confer greater overall metapopulation resilience to threats in agricultural land. However, if this results in carnivore population growth, any concomitant increase in livestock depredation would likely prompt rising persecution of suspected culprits (Inskip and Zimmermann, 2009; Treves and Karanth, 2003). In view of this, programmes that support farmers in implementing non-lethal anti-predation methods may also be increasingly relevant. 
Our simulation results provide a good starting point for designing studies that balance precision, bias and power, where the aim of a survey is to monitor both occupancy and abundance from detection/non-detection data. We found that six replicates per site is adequate for estimating brown hyaena occupancy. With $>5$ replicates, the Royle-Nichols model performs reasonably well at sample sizes $\leqslant 100$, especially if detection probability is $\geqslant 0.3$ (Royle and Nichols, 2003). This may often be achievable in carnivore sign surveys. For example, a North American study estimated detection probability from snow tracking of coyotes (Canis latrans), fishers (Martes pennanti) and domestic cats (Felis catus) at $>0.3,>0.4$ and $>0.5$ respectively (Gompper et al., 2006). However, Hines et al. $(2010)$ report tiger detection probability at $\leqslant 0.14$ in their sign survey, and for such rare species, $\geqslant 10$ replicates or sample sizes $>100$ are recommended (Royle and Nichols, 2003).

Increasing effort to 12 replicates improved RMSE and CV, which would otherwise be unacceptably high for our brown hyaena abundance estimates. Furthermore, adding replicates is likely to be relatively efficient in a cluster-sampling context because once observers have reached a site, the cost of performing additional sign survey replicates is low (Mackenzie and Royle, 2005). In addition, other authors have suggested that completing more replicates rather than increasing sample size is particularly efficient for species with high occupancy (Field et al., 2005; Mackenzie and Royle, 2005; Mattfeldt et al., 2009). That being said, power increases with sample size. Other carnivore studies have used monitoring criteria of $\alpha=\beta=0.2$ (Barlow et al., 2008), which assumes equal economic, social, political and environmental costs of Type I and II errors (Mapstone, 1995). Using these criteria, annual effort of 12 replicates at each of a simple random sample of $\geqslant 65$ sites would offer sufficient power to detect a $50 \%$ decline in brown hyaena occupancy in our study area over a 5 year period. It would also produce abundance estimates with acceptable bias and precision, especially if the survey can be designed to minimise missing observations and avoid the associated loss of precision. One way of achieving this would be to account for variation in the proportion of agricultural land in the site by modelling it as a covariate rather than scaling effort.

A single survey of $\geqslant 65$ sites would provide unbiased and reasonably precise brown hyaena population parameter estimates. However, the aim of many monitoring programmes is to provide early warning that management or recovery actions are needed (Field et al., 2005). We cannot yet determine the effort or timescale required to detect a $10 \%$ decline in brown hyaena abundance, but waiting 5 years to detect a $50 \%$ occupancy decline for a low density species is probably unacceptably risky. Surveying over a longer period may enable detection of smaller trends but sampling a larger number of sites would be a safer strategy. Whereas it may be impractical to survey more than 75 sites using our sampling frame in our study area, it would be appropriate in surveys with similar extent but smaller grid cell dimensions, or similar grid cell dimensions and a larger extent. For example, brown hyaenas have larger home ranges than most sympatric species, so smaller grid cells could be used for other carnivores, increasing the practicality of larger samples without increasing the extent of the survey. For wide-ranging species, it may be reasonable to propose an annual survey of $>200$ grid cells across a larger study area, especially if conservation organisations solicit help from land owners. This approach is illustrated by other large-scale volunteer wildlife monitoring programs, such as the UK Breeding Bird Survey (Risely et al., 2010). Thousands of volunteers participate nationwide, producing sufficient data to monitor population trends in many UK bird species. Such surveys might also enhance public awareness of carnivore ecology, potentially improving attitudes to carnivores as well as providing sufficient power to detect small effect sizes.

\section{Acknowledgements}

We thank K.U. Karanth for inspiring an occupancy-based approach, and the authors of Hines et al. (2010) for providing details of their sampling design ahead of publication, and helping with queries concerning data analysis. We thank one anonymous reviewer and M. Linkie for helpful comments on the manuscript. We are indebted to the private land-owners whose land we surveyed and to $S$. Uzzell for the computer equipment he provided. We are also grateful to the University of Brighton, UK, the Earthwatch Institute and the Leverhulme Trust, UK for funding.

\section{References}

Bailey, L., Hines, J.E., Nichols, J.D., MacKenzie, D.I., 2007. Sampling design trade-offs in occupancy studies with imperfect detection: examples and software. Ecological Applications 17, 281-290.

Barlow, A.C.D., Ishtiaq, M.A.U., Rahman, M.M., Howlader, A., Smith, A.C., Smith, J.L.D., 2008. Linking monitoring and intervention for improved management of tigers in the Sundarbans of Bangladesh. Biological Conservation 141, 20322040.

Burnham, K.P., Anderson, D.R., 2002. Model Selection and Multimodel Inference: A Practical Information-theoretic Approach, second ed. Springer-Verlag, New York.

Burnham, K.P., Anderson, D.R., White, G.C., Brownie, C., Pollock, K.P., 1987. Design and analysis of methods for fish survival experiments based on releaserecapture. American Fisheries Society Monograph 5, 1-437.

Donovan, T.M., Hines, J.E., 2007. Exercises in Occupancy Modeling and Estimation. <http://www.uvm.edu/envnr/vtcfwru/spreadsheets/occupancy.htm>.

Field, S.A., Tyre, A.J., Possingham, H.P., 2005. Optimizing allocation of monitoring effort under economic and observational constraints. Journal of Wildlife Management 69, 473-482.

Friedmann, Y., Daly, D. (Eds.), 2004. Red Data Book of the Mammals of South Africa: A Conservation Assessment. CBSG Southern Africa, Conservation Breeding Specialist Group (SSC/IUCN) \& The Endangered Wildlife Trust, Johannesburg.

Gompper, M.E., Kays, R.W., Ray, J.C., Lapoint, S.D., Bogan, D.A., Cryan, J.R., 2006. A comparison of noninvasive techniques to survey carnivore communities in northeastern North America. Wildlife Society Bulletin 34, 1142-1151.

Hejcmanova, P., Hejcman, M., Camara, A.A., Antoninova, M., 2010. Exclusion of livestock grazing and wood collection in dryland savannah: an effect on longterm vegetation succession. African Journal of Ecology 48, 408-417.

Hines, J.E., 2006. PRESENCE 2.3 - Software to Estimate Patch Occupancy and Related Parameters. USGS-PWRC. <http://www.mbr-pwrc.usgs.gov/software/ presence.html>

Hines, J.E., Nichols, J.D., Royle, J.A., MacKenzie, D.I., Gopalaswamy, A.M., Samba Kumar, N., Karanth, K.U., 2010. Tigers on trails: occupancy modeling for cluster sampling. Ecological Applications 20, 1456-1466.

Hoffman, T., Ashwell, A., 2001. Nature Divided: Land degradation in South Africa. University of Cape Town, Cape Town.

Inskip, C., Zimmermann, A., 2009. Human-felid conflict: a review of patterns and priorities worldwide. Oryx 43, 18-34.

Karanth, K.K., Nichols, J.D., Hines, J.E., Karanth, U.K., Christensen, N.L., 2009. Patterns and determinants of mammal species occurrence in India. Journal of Applied Ecology 46, 1189-1200.

Karanth, K.U., Nichols, J.D. (Eds.), 2002. Monitoring Tigers and Their Prey: A Manual for Researchers, Managers and Conservationists in Tropical Asia. Centre for Wildlife Studies, Bangalore.

Linkie, M., Dinata, Y., Nugroho, A., Haidir, I.A., 2007. Estimating occupancy of a data deficient mammalian species living in tropical rainforests: Sun bears in the Kerinci Seblat region, Sumatra. Biological Conservation 137, 20-27.

Linkie, M., Guillera-Arroita, G., Smith, J., Rayan, M.D., 2010. Monitoring tigers with confidence. Integrative Zoology 5, 342-350.

MacKenzie, D.I., Bailey, L.L., 2004. Assessing the fit of site-occupancy models Journal of Agricultural Biological and Environmental Statistics 9, 300-318.

MacKenzie, D.I., Nichols, J.D., Lachman, G.B., Droege, S., Royle, J.A., Langtimm, C.A., 2002. Estimating site occupancy rates when detection probabilities are less than one. Ecology 83, 2248-2255.

MacKenzie, D.I., Nichols, J.D., Royle, J.A., Pollock, K., Bailey, L., Hines, J.E., 2006 Occupancy Estimation and Modelling - Inferring Patterns and Dynamics of Species Occurrence. Elsevier Publishing, London.

Mackenzie, D.I., Royle, J.A., 2005. Designing occupancy studies: general advice and allocating survey effort. Journal of Applied Ecology 42, 1105-1114.

Mapstone, B., 1995. Scalable decision rules for environmental impact studies: effect size, Type I and Type II errors. Ecological Applications 5, 401-410.

Mattfeldt, S.D., Bailey, L.L., Grant, E.H.C., 2009. Monitoring multiple species: estimating state variables and exploring the efficacy of a monitoring program. Biological Conservation 142, 720-737.

Maude, G., 2005. The Comparative Ecology of the Brown Hyaena (Hyaena brunnea) in Makgadikgadi National Park and a Neighbouring Community Cattle Area of Botswana, In Department of Zoology and Entomology. University of Pretoria, Pretoria. 
Mills, M.G.L., 1982. The mating system of the brown hyaena, Hyaena brunnea in the southern Kalahari. Behavioral Ecology and Sociobiology 10, 131-136.

Risely, K., Baillie, S.R., Eaton, M.A., Joys, A.C., Musgrove, A.J., Noble, D.G., Renwick, A.R., Wright, L.J., 2010. The Breeding Bird Survey 2009. BTO Research Report 559. British Trust for Ornithology, Thetford.

Royle, J.A., Nichols, J.D., 2003. Estimating abundance from repeated presenceabsence data or point counts. Ecology 84, 777-790.

Sewell, D., Beebee, T., Griffiths, R.A., 2010. Optimising biodiversity assessments by volunteers: the application of occupancy modeling to large-scale amphibian surveys. Biological Conservation 143, 2102-2110.

Stuart, C., Stuart, T., 2000. A field guide to the tracks and signs of southern and East African wildlife. Struik, Cape Town.

Thorn, M., Green, M., Bateman, P., Cameron, E.Z., Yarnell, R., Scott, D., 2010. Comparative performance of sign surveys, spotlighting, and audio playbacks in a landscape-scale carnivore survey. South African Journal of Wildlife Research $40,77-86$.

Thorn, M., Scott, D.M., Green, M., Bateman, P.W., Cameron, E.Z., 2009. Estimating brown hyaena occupancy using baited camera traps. South African Journal of Wildlife Research 39, 1-10.

Tladi, B., Baloyi, T., Marfo, C., 2002. Chapter 6: settlement and land use patterns. In: Walmsley, D., Walmsley, J., Mangold, S., Kalule-Sabiti, M. (Eds.), North West
Province State of the Environment Report. Directorate of Environment and Conservation Management. North West Department of Agriculture, Conservation and Environment, Mmabatho.

Treves, A., Karanth, K.U., 2003. Human-carnivore conflict and perspectives on carnivore management worldwide. Conservation Biology 17, 1491-1499.

Watson, C.A., Weckerly, F.W., Hatfield, J.S., Farquhar, C.C., Williamson, P.S., 2008. Presence-nonpresence surveys of golden-cheeked warblers: detection, occupancy and survey effort. Animal Conservation 11, 484-492.

White, G.C., Burnham, K.P., 1999. Program MARK: survival estimation from populations of marked animals. Bird Study 46, 120-139.

Wiesel, I., Maude, G., Scott, D., Mills, G., 2008. Hyaena brunnea, In 2008 IUCN Red List of Threatened Species. <www.iucnredlist.org>.

Woodroffe, R., Ginsberg, J.R., 1998. Edge effects and the extinction of populations inside protected areas. Science 280, 2126-2128.

Zeller, K.A., Nijhawan, S., Salom-Pérez, R., Potosme, S.H., Hines, J.E., 2011. Integrating occupancy modeling and interview data for corridor identification: a case study for jaguars in Nicaragua. Biological Conservation 144, 892-901.

Zimmermann, A., Walpole, M.J., Leader-Williams, N., 2005. Cattle ranchers' attitudes to conflicts with jaguar Panthera onca in the Pantanal of Brazil. Oryx 39, 406-412. 